\title{
Fractal and Transgenerational Genetic Effects on Phenotypic Variation and Disease Risk
}

\author{
Joe Nadeau \\ Institute for Systems Biology
}

To understand human biology and to manage heritable diseases, a complete picture of the genetic basis for phenotypic variation and disease risk is needed. Unexpectedly however, most of these genetic variants, even for highly heritable traits, continue to elude discovery for poorly understood reasons. The genetics community is actively exploring the usual explanations for missing heritability. But given the extraordinary work that has already been done and the exceptional magnitude of the problem, it seems likely that unconventional genetic properties are involved.

We made two surprising discoveries that may dramatically change our understanding of the genetic basis for phenotypic variation and disease risk, and that may also explain much of missing heritability. The first property involves fractal genetics, where a very large number of often closely linked genetic variants act in a remarkably strong, non-additive and context-dependent manner to control phenotypic variation, suggesting that networks of gene interactions are more important than the constant action of individual variants. The second property involves transgenerational genetic effects, where genetic variants acting in one generation affect phenotypes in subsequent generations. Because these transgenerational effects are common, strong and persistent across multiple generations, they rival the action of genetic variants that are inherited in the conventional manner. The search is ongoing to identify the molecular basis for this non-DNA inheritance. Together these discoveries in model organisms shed light on genetic phenomena that impact human biology but that are difficult to extremely detect directly in human populations. In particular, inheritance of traits and diseases without the corresponding genetic variants could revolutionize our understanding of inheritance. 